\title{
Atividade física e envelhecimento humano: a busca pelo envelhecimento saudável
}

Mauro Lúcio Mazini Filho*, André Luiz Zanella*, Felipe José Aidar*, Aurélio Meirelles Soares da Silva*, Rosimar da Silva Salgueiro"*", Dihogo Gama de Matos"***

\section{Resumo}

O envelhecimento populacional vem tomando proporções significativas nos últimos trinta anos em nosso país. A expectativa é a de que no Brasil o número de pessoas idosas irá se igualar aos países europeus, tornando-nos o sexto país com o maior número de idosos até o ano de 2025. A melhoria na qualidade de vida e o aumento da expectativa de vida levam a que o número de pessoas que atingirão a terceira idade irá aumentar significativamente em nosso país. Dentre os diversos fatores que têm contribuído para esse fenômeno estão a preocupação com o estilo de vida e o incremento da atividade física. Assim, o objetivo deste estudo foi contribuir com a literatura, informando como o processo de envelhecimento evolui no corpo humano e como a atividade física pode intervir no envelhecimento populacional. Para isso foi realizado um estudo descritivo com uma análise qualitativa sobre envelhecimento e atividade física nos idosos. Concluímos que não se pode interferir no processo de envelhecimento mesmo com a prática regular de atividade física, mas que esta pode auxiliar no processo evolutivo do envelhecimento, conduzindo a que a manifestação dos sintomas do envelhecimento e o surgimento de doenças, como a hipertensão e as doenças cardiovasculares, sejam reduzidos, o que, por conseqüência, diminuirá também o consumo de remédios.

Palavras-chave: Envelhecimento. Idosos. Atividade física.

\section{Introdução}

O envelhecimento populacional vem tomando proporções significativas primordialmente nos últimos trinta anos, visto que países em desenvolvimento como o Brasil, segundo as projeções estatísticas da Organização Mundial de Saúde (WHO, 2004), bem como do

* Acadêmicos do Programa de Pós-Graduação em Avaliação das Actividades Física e Desportivas pela Universidade de Trás-os-Montes e Alto Douro, Vila Real - Portugal.

** Acadêmico do curso de Especialização em Saúde da Família da Universidade do Estado do Pará.

*** Graduados em Educação Física pela Universidade Federal de Juiz de Fora.

***** Mestre em Educação Física e Desporto com ênfase em Avaliação das Actividades Físicas e Desportivas pela Universidade de Trás-os-Montes e Alto Douro, Vila Real - Portugal. Professor da Universidade Federal de Juiz de Fora. Endereço para correspondência: Rua Jornalista Carlos Tito, 40. CEP: 25.811-160. Três Rios - RJ. E-mail: dihogogmc@hotmail.com.

$\hookrightarrow$ Recebido em agosto de 2009 - Avaliado em março 2010.

$\hookrightarrow$ doi:10.5335/rbceh.2010.010 
Instituto Brasileiro de Geografia e Estatística (IBGE, 2004), entre 1950 e 2025 a população de idosos crescerá dezesseis vezes contra somente cinco vezes da população total. A proporção passará de, aproximadamente, $7,5 \%$ para $15 \%$, que é a mesma de países europeus. Esse fato coloca o Brasil, em termos absolutos, como o sexto país em idosos no mundo.

A expectativa de vida tem aumentado muito em função de inúmeros fatores, tais como o avanço da medicina, as novas técnicas de fisioterapia, os maiores conhecimentos na área de nutrição, as informações que a mídia constantemente veicula, o treinamento físico propriamente dito, dentre muitos outros. (BEMBEM, 1998). Além disso, a diminuição da taxa de natalidade, juntamente com a diminuição da taxa de mortalidade, deve ser levada em consideração para que tal fato realmente aconteça. (WHO, 2004).

Pode-se entender o envelhecimento como um processo progressivo e inevitável, caracterizado pela diminuição das funções fisiológicas e de todas as capacidades físicas, o qual torna essa população dependente de terceiros para que conseguir realizar as atividades cotidianas. (MURRAY; LOPES, 1997).

Conhecendo essas informações, Chakravarthy, Joyner e Booth (2002) afirmam que a prescrição de atividades físicas combinadas para promoção da saúde visa combater o sedentarismo, que é o grande aliado das causas de doenças ocorridas com o envelhecimento.

Assim, o objetivo do presente estudo é, por meio de uma revisão de literatura, expor os benefícios e efeitos da atividade física no processo de envelhecimento.

\section{Envelhecimento}

O envelhecimento é um processo progressivo e inevitável, caracterizado pela diminuição das funções fisiológicas e de todas as capacidades físicas. (MURRAY; LOPES, 1997). Alterações na saúde mental, na cognição e no humor também são comuns na população idosa. (COSTIL, 1986).

Pesquisas de Heath et al. (1981) demonstram que, com o passar dos anos, ocorrem perdas naturais das capacidades físicas, como a perda de força, de flexibilidade, de velocidade, dos níveis de $\mathrm{VO}_{2}$, de massa óssea (osteopenia), além da redução na massa muscular (sarcopenia), em razão, sobremaneira, do acometimento nas fibras do tipo IIb. Além disso, nessa fase ocorrem o aumento da gordura corporal e o aparecimento de várias patologias decorrentes das mudanças citadas, como, por exemplo, a osteoporose, doenças cardiovasculares, diabetes, hipertensão arterial, dentre outras.

Essas alterações fisiológicas e musculoarticulares, associadas à inatividade física, levam, geralmente, o idoso a uma condição degenerativa crescente de suas capacidades físicas e fisiológicas, acarretando o aparecimento de problemas como a perda de equilíbrio (ataxia), o comprometimento da marcha e os problemas psicológicos como a baixa estima e depressão. (MATSUDO, 2002). Tendo isso em vista, é sugerido na literatura que grande parte desses aspectos deletérios do envelhecimento pode ser amenizada com a intervenção por meio da prática de exercícios físicos regulares. (WEINECK, 2005). 
De acordo com Powers e Howley (2000), podemos evidenciar duas principais teorias para o envelhecimento biológico: uma propondo uma explicação genética, entendendo que o envelhecimento aparece como tendo sido programado biologicamente; outra afirmando que os radicais livres promovem a peroxidação da membrana lipídica, a qual pode interferir em muitos processos celulares. (COSTIL, 1986). O posicionamento em relação ao conceito do processo da velhice é divergente em vários autores, o que deixa clara a grande dificuldade para a compreensão desse estágio da vida. (KAMEN et al., 1995; JORFELDT; WHAREN, 1971).

Envelhecer é uma fase complexa e que envolve muitas variáveis que interagem entre si, influenciando grandemente na maneira como se envelhece. (GOODRICK, 1980). Transformações nos parâmetros sociais e ambientais irão ocorrer, pois o idoso passa a vivenciar dificuldades quanto à sua segurança $\mathrm{e}$ à adequação do ambiente em que vive. (ROBINSON, 1938).

Portanto, com o aumento da população idosa mundial, uma das grandes preocupações nos países desenvolvidos é a questão da incapacidade dos sujeitos idosos e os possíveis custos que esse fato pode causar, pois com o envelhecimento é natural que ocorra um aumento na dependência. (MATSUDO, 2002). Por isso, manter essa população ativa com a prática de hábitos de vida saudáveis, como alimentação balanceada, exercícios regulares e atividades sociais, pode ser alternativa eficaz na diminuição dos gastos dos cofres públicos e na melhoria da saúde dessa população. (SALTIN, 1990).
O processo de envelhecimento populacional em países desenvolvidos ocorreu de forma gradual e acompanhado de melhorias na cobertura do sistema de saúde, nas condições de habitação, saneamento básico, trabalho e alimentação. No Brasil ocorre rapidamente e em um contexto de desigualdades sociais, economia frágil, níveis de pobreza, precário acesso aos serviços de saúde e reduzidos recursos financeiros, ou seja, esse processo ocorre sem que haja modificações estruturais que respondam às demandas do novo grupo etário emergente. (GARRETT et al., 2004; CHAKRAVARTHY; JOYNER; BOOTH, 2002).

\section{Exercício físico e envelhecimento}

Os benefícios da atividade física para a saúde e longevidade são intuitivamente conhecidos desde o princípio dos tempos, existindo benefícios bem demonstrados sobre vários parâmetros que afetam a saúde e a longevidade. (CORAZZA, 2001). Muitos trabalhos, como o proposto por Burns et al. (2003), enfatizam a importância da ação dos profissionais de saúde e entidades governamentais no estímulo à atividade física, assim como seu impacto sobre a saúde pública.

Apesar de existir uma associação entre atividade física e saúde, a maior parte da população é inativa completa ou parcialmente, visto que nas últimas décadas, com o fenômeno da urbanização de nossas cidades e sociedades, nosso estilo de vida tornou-se menos ativo. (MAZO; BENEDETTI; LOPES, 2004). Segundo eles, podem existir riscos e barreiras individuais relacionados aos 
exercícios; desse modo, a prática de atividade física e de exercícios deve ser prescrita individualmente, principalmente entre os idosos.

O envelhecimento populacional é uma realidade no nosso país, assim como em todo o mundo. Assim, conforme Burns et al. (2003), com o aumento do número de idosos aumentam as doenças associadas ao envelhecimento. A dependência é o problema que mais afeta a qualidade de vida dos idosos, tanto para realizar as atividades de vida diária quanto as atividades instrumentais de vida diária, o que pode ser consequência de doenças neurológicas, cardiovasculares, fraturas, lesões articulares, entre outras. (MATSUDO, 2002).

Para Benestad (1965), a atividade física regular pode contribuir muito para evitar os problemas trazidos pelo envelhecimento, e seu enfoque principal deverá ser a promoção de saúde. Contudo, em indivíduos com patologias já instaladas a prática de exercícios orientados pode ser muito importante para controlar a doença, evitar sua progressão e/ou reabilitar o paciente.

Child, Barnard e Taw (1984) afirmam que chegar à fase de "envelhecimento" bem e ativo fisicamente são conceitos fortemente associados para pessoas que já passaram dos quarenta anos de idade, sendo incentivadas por professores de educação física, fisioterapeutas, médicos e psicólogos. A prática constante e moderada de exercícios é um excelente método de melhoria da qualidade de vida dessas pessoas, seja em modalidades especiais, seja usando o fluxo de oportunidades do seu dia a dia.

\section{0 efeito do envelhecimento nas variáveis fisiológicas}

À medida que envelhecemos, os desempenhos máximos, tanto nos eventos de endurance quanto nos de força, diminuem aproximadamente $1 \%$ a $2 \%$ ao ano, iniciando em torno dos $20 \mathrm{e}$ 35 anos. (DRINKWATER; HORVATH; WELLS, 1975).

Powers e Howley (2000) afirmam que a maior parte dos desempenhos atléticos declina numa taxa constante durante a meia idade e a velhice, em decorrência, sobretudo, das diminuições da resistência muscular e cardiovascular e da força. $\mathrm{O} \mathrm{VO}_{2 \text { máx, }}$ por exemplo, diminui aproximadamente $10 \%$ por década com o envelhecimento, iniciando no final da adolescência, para as mulheres, e em meados da segunda década de vida, para os homens. Essa diminuição está, em grande parte, associada a uma diminuição da atividade de resistência cardiovascular.

É muitas vezes difícil diferenciar entre os resultados do envelhecimento biológico e os da inatividade física. Com o envelhecimento, ocorre uma deterioração natural da função fisiológica, a qual se deve ao fato de a maioria das pessoas se tornar mais sedentária à medida que envelhece. (CRESS et al., 1995).

Tanto a capacidade vital quanto o volume expiratório forçado diminuem linearmente com a idade. O volume residual aumenta e a capacidade pulmonar total permanece inalterada, significando que menos ar pode ser trocado em cada respiração. (MATSUDO, 2002).

As alterações pulmonares que acompanham o envelhecimento são causadas 
principalmente pela perda de elasticidade do tecido pulmonar e da parede torácica. Porém, as pessoas mais velhas apresentam somente uma discreta diminuição da capacidade pulmonar total. Para elas, o principal limitador do $\mathrm{VO}_{2 \text { máx }}$ seria a diminuição do transporte de oxigênio aos músculos. (POWERS; HOWLEY, 2000). Cress et al. (1995), entretanto, afirmam que essas alterações são minimizadas nos atletas mais velhos que continuam a treinar, o que indicaria que a inatividade pode ter um papel maior do que o envelhecimento físico.

A força também é afetada e reduzida com o envelhecimento. Isso ocorre em consequência das limitações tanto da atividade física quanto da massa muscular, que seria o resultado de uma redução da síntese protéica ocorrida com o envelhecimento e da perda de unidades motoras das fibras de contração rápida. Enquanto o treinamento de endurance tem pouco impacto na prevenção da perda de massa muscular com o envelhecimento, o treinamento de força pode manter ou aumentar a área transversa das fibras musculares de homens e mulheres mais velhos. (OLIVEIRA; TROCOLLI, 2000).

Segundo Powers e Howley (2000), em geral as pessoas normalmente ativas apresentam um desvio em direção a uma maior porcentagem de fibras musculares de contração lenta à medida que envelhecem, em virtude de uma redução da quantidade de fibras de contração rápida.

O envelhecimento também diminui a capacidade do sistema nervoso de detectar um estímulo e de processar informações para produzir uma resposta.
O treino não consegue interromper o processo de envelhecimento biológico, mas pode reduzir o seu impacto sobre o desempenho. (MATSUDO, 2002).

\section{Benefícios da atividade física no envelhecimento}

A atividade física é um ponto importante no processo de envelhecimento. No entanto, o tipo de exercício a ser realizado depende do organismo e da vontade de cada indivíduo. Assim, Costil (1986) informa que não há nenhuma fórmula predeterminada do que deve ser feito na terceira idade. Segundo o autor, com o auxílio do profissional de saúde o idoso precisa olhar para si e ver qual é a sua capacidade funcional nas atividades do dia a dia, como subir as escadas de um ônibus, carregar panelas de pressão, arrumar camas, abaixar-se para ver o forno. Assim, a melhora da atividade física melhorará, posteriormente, sua capacidade de desempenhar essas e outras tarefas cotidianas.

Cress (1995) afirma que a prática de atividade física regular e sistemática aumenta ou mantém a aptidão física das pessoas idosas, com o potencial de aumentar o seu bem-estar funcional e, consequentemente, diminuir a taxa de morbidade e de mortalidade entre essa população, doenças coronarianas, a hipertensão, o diabetes insulino não dependentes, a hiperlipidemia (lipídeos elevado no sangue). Essas poderão ter seus efeitos controlados pela prática de exercícios físicos, além de se prevenir ou retardar essas doenças, aumentando, assim, a expectativa de vida para essa população. 
Para Kamen et al. (1995), os benefícios do exercício podem ter grande impacto sobre a saúde pública, porém deve estar inserido dentro de um programa global de intervenção sobre os fatores de risco cardiovasculares, com avaliação médica. A atividade física deve ser realizada em ambiente supervisionado por profissional de saúde, apto a monitorar a frequência cardíaca, pressão arterial e os sintomas que se manifestarem durante a prática.

Dentre os benefícios da atividade física, Matsudo (2002) cita os que seriam os mais importantes: melhora da sensibilidade à insulina, levando a um melhor controle glicêmico, que pode prevenir o desenvolvimento de diabetes, aumento da fração HDL, diminuição da LDL, redução significativa dos triglicérides. Com o envelhecimento ocorre um aumento percentual da gordura corporal e diminuição da massa muscular, porém a atividade física reduz essas modificações. Além disso, ajuda a melhorar a massa óssea quando jovem e prevenir a perda na fase adulta, diminuindo o risco de fraturas; melhora a imunidade, que pode diminuir a incidência de infecções e, possivelmente, de certos tipos de câncer; melhora a função autonômica, com aumento da sensibilidade dos baroreceptores e da variabilidade da frequência cardíaca.

Ainda segundo Jorfeldt e Wahren (1971), um dos benefícios mais bem documentados é sobre o risco de doença coronariana e morte, havendo uma relação inversa com a prática de exercício habitual, o que é demonstrado tanto para a prática de exercício programado quanto para as atividades de lazer ou inseridas nas rotinas do dia a dia. Weineck (2005) finaliza demonstrando em seu estudo que, apesar de o exercício moderado já apresentar benefícios, aparentemente existe uma relação dose-resposta, com exercícios mais vigorosos demonstrando um efeito ainda maior se comparados com exercícios mais intensos.

\section{Riscos da atividade física no envelhecimento}

Na visão de Bemben (1998), como o envelhecimento é um processo heterogêneo, a recomendação de atividade física para os idosos deve ser individualizada. Para o autor, os benefícios da atividade física no envelhecimento podem ser observados pelas respostas fisiológicas do organismo, além de apresentar um efeito favorável sobre o equilíbrio e a marcha, diminuindo o risco de quedas e fraturas, menor dependência para realização de atividades de vida diária, melhora na autoestima e autoconfiança, elevando de forma significativa à qualidade de vida.

Matsudo (2002) afirma que, ao trabalhar com idosos, precisamos fazer uma avaliação médica pré-exercício, necessária porque o nível de atividade física que é considerada vigorosa para 0 idoso não pode ser idêntico ao do adulto saudável. Segundo o autor, esforços de intensidade moderada já podem ser considerados como vigorosos para um idoso sedentário, mesmo que considerado apto em uma avaliação inicial.

Pollock et al. (1997) ressaltam que uma avaliação médica inicial é obrigatória para todos os homens com mais 
de quarenta anos, mulheres com mais cinquenta anos, portadores de doenças crônicas (tal como doença coronariana, pulmonar, diabetes) ou que possuem fatores de risco cardiovasculares (diabetes, hipertensão, tabagismo e outros). Ainda, todos os indivíduos com sessenta anos ou mais devem ser submetidos à avaliação médica periódica e o clínico ou geriatra que o acompanha deve estar apto a liberar e recomendar a atividade física.

A atividade física é um importante fator na melhoria do envelhecimento e qualidade de vida dos idosos, desde que os princípios científicos e o bom senso sejam considerados durante todo o processo de treinamento do indivíduo. (SALTIN, 1990).

Pollock et al. (1997) referem que a prática de qualquer atividade física deve seguir uma frequência longitudinal no cotidiano do indivíduo, sendo realizada ao longo de todo o seu desenvolvimento, desde a juventude, para que a gama de benefícios dela advindos seja ainda mais otimizada, o que se refletirá em uma vida muito mais saudável, independente e duradoura.

A atividade física regular pode contribuir muito para evitar as incapacidades associadas ao envelhecimento. Seu enfoque principal deve ser a promoção de saúde, porém em indivíduos com patologias já instaladas a prática de exercícios orientados pode ser muito importante para controlar a doença, evitar sua progressão e/ou reabilitar o paciente. (ROBINSON, 1938).

Dentre alguns estudos encontrados o que nos chamou maior atenção foi o de Silva e Barros (2000), o qual afirma que com a redução na prática de atividade física vem ocorrendo um aumento na prevalência de obesidade juntamente com o envelhecimento. Para os autores, quanto maior a idade, menor a chance do indivíduo de estar engajado em alguma atividade física regular; ainda, pelo menos $70 \%$ dos idosos têm algum problema de saúde, e a atividade física poderia ser uma grande aliada do tratamento para diminuir tais números.

Finalizando e concordando com as afirmações supracitadas, Matsudo (2002) defende que a prática da atividade física pode controlar a manifestação e os sintomas de várias doenças, como a hipertensão, e também reduzir o consumo de remédios. Para isso, seria preciso trabalhar com três sistemas do corpo humano: o cardiovascular, o nervoso e o musculoesquelético.

\section{Conclusão}

Diante dessa revisão, é possível concluir que é muito importante a prática regular de exercícios físicos não apenas na juventude, mas, sim, ao longo de toda uma vida. A prática constante de algum tipo de exercício físico mostrase importante na medida em que pode proporcionar um aumento na qualidade de vida do idoso, auxiliar na manutenção da capacidade funcional do idoso e na manutenção plena das habilidades físicas e mentais que adquiriu ao longo de sua vida.

O que não podemos deixar de salientar, que foi praticamente uma unanimidade dentre os autores consultados, é que não se pode alterar o processo de 
envelhecimento humano, porém esse é influenciado pela inatividade física. Portanto, a prática constante de atividade física é atua como meio de controlar o surgimento de sintomas de várias doenças, como a hipertensão, doenças cardiovasculares e doenças musculoesqueléticas.

A idade pareceu não comprometer a capacidade da pessoa para aumentar a força muscular ou a hipertrofia muscular, porém se desenvolve em um ritmo lento em razão das mudanças fisiológicas e endócrinas ocorridas com o organismo.

A atividade física regular pode ser considerada, portanto, como o contribuinte mais importante para a boa saúde. Por isso, pode-se dizer que a atividade física contribui para a longevidade na vida adulta, visto que a diminuição da força e da resistência aeróbia em homens e mulheres é causada, sobretudo, por uma redução de freqüência cardíaca e do volume de ejeção cardíaco, o que provoca a limitação do transporte de oxigênio aos tecidos.

Não podemos deixar de destacar também que existem várias teorias sobre o envelhecimento, mas infelizmente algumas delas são difíceis de serem comprovadas, exigindo maiores pesquisas e investimentos. Contudo, com nosso estudo de revisão pode-se dizer que fatores como o sexo e o estilo de vida das pessoas são fortemente influenciados pela prática ou não de atividades físicas. Cabe, portanto, ao profissional da área da saúde conscientizar-se e prevenir o processo de envelhecimento por meio de algum método de trabalho, favorecendo e estimulando a prática constante de exer- cícios físicos para, assim, proporcionar um envelhecimento saudável.

Physical activity and human aging: the search for healthy aging

\section{Abstract}

The old age population come capture meaningful proportions, overridingly us bottom 30 years in ours country, where the anticipation be the of us equalize the Europe, us assume in this country of elderly to 2025 . With the best in the grade life, the life expectancy and him act of peoples that attain the third age tendency at accrue. Dentures them sundry factors that have contribute to this phenomenon be, definitely, at care coat bodily life style and the increment of the activity. In The Circumstances the object give study be the of advance active the old age exhibit the benefits of the activity bodily no process of old age, where be fulfilled a study descriptive with qualitative analysis of the advancement of him old age and of the figures of avert e develop awareness the aged of the amount of the activity before e during the best age. Concluding whether hence that be unable to interfere no only lawsuit of old age be able to belittle with bodily average the observance of activity, so that be able to check the demonstration and the symptoms of sundry diseases, as the hypertension and the diseases cardiac asset consume decrease the consumption of drugs in the search coat old age activate buxom.

Key words: Aging. Elder. Motor activity. 


\section{Referências}

BEMBEM, M. G. Age-related alterations in muscular endurance. Sports Medicine, v. 25, n. 4, p. 259-269, 1998.

BENESTAD, A. Trainability of old men. Acta Medica Scandinavica, v.178, s. n, p. 321, 1965.

BURNS, V. R. N. R. et al. Estudo comparativo da flexibilidade em mulheres idosas no teste de sentar e alcançar antes e depois de uma sessão de atividade física. In: SIMPÓSIO INTERNACIONAL DE CIÊNCIA DO ESPORTE, XXVI, 2003, São Paulo. Anais... São Paulo: Celafiscs, 2003.

CHAKRAVARTHY, M. V; JOYNER, M. J.; BOOTH, F. W. An obligation for primary care physicians to prescribe physical activity to sedentary patients to reduce the risk of chronic health conditions. Mayo Clin. Proc., v. 77, s. n, p. 165-73, 2002.

CHILD, J. S.; BARNARD, R. J.; TAW, R. L. Cardiac hypertrophy and function in master endurance runners and sprinters. Journal of Applied Physiology: Respiratory, Environmental, and Exercise Physiology, v. 57, s. n., p. 176-181, 1984.

CORAZZA, M. A. Terceira idade: atividade física. São Paulo: Phorte, 2001.

COSTIL, D. L. Inside running: basics of sports physiology. Indianapolis: Benchmark Press, 1986.

CRESS, M. E. et al. Relationship between physical performance and self-perceived physical function. J. Am. Geriatric. Soc., v. 43, n. 2 , p. $93-101,1995$.

DRINKWATER, B. L.; HORVATH, S. M.; WELLS, C. L. Aerobic power of females, ages 10 to 68. Journal of Gerontology, v. 30, n. 4, p. 385-394, 1975.

GARRETT, N. A. et al. Physical inactivity: direct cost to a health plan. Am. J. Prev. Med., v. 27, n. 5, p. 304-309, 2004.
GOODRICK, C. L. Effects of long-term voluntary wheel exercise on male and female wistar rats: 1 . Longevity, body weight and metabolic rate. Gerontology, v. 26, s. n, p. 22-33, 1980.

HEATH, G. W. et al. A physiological comparison of young and older endurance athletes. Journal of Applied Physiology: Respiratory, Environmental, and Exercise Physiology, v. 51, s. n, p. 634-640, 1981.

IBGE - Instituto Brasileiro de Geografia e Estatística. Projeção de população do Brasil por sexo e idade para o período de 198020050. Diretoria de Pesquisa. Coordenação de População e Indicadores Sociais. Rio de Janeiro: IBGE, 2004.

JORFELDT, L.; WAHREN, J. Leg blood flow during exercise in man. Clinical Science, v. 41, s. n, p. 459-473, 1971.

KAMEN, G. et al. Motor unit discharge behavior in older adults during maximal-effort contrations. Journal of Applied Physiology, v. 79, s. n, p. 1908-1913, 1995.

MAZO, G. Z.; BENEDETTI, T. B.; LOPES, M. A. Atividade física e o idoso. Porto Alegre: Sulina, 2004.

MATSUDO, S. M. Envelhecimento, atividade física e saúde. Revista Mineira de Educação Física, Viçosa, v. 10, n. 1, p. 193-207, 2002.

MURRAY, C. J.; LOPEZ, A. D. Mortality by cause for eight regions of the world: global burden of disease study. Lancet, v. 349, n. 9061, p. 1269-1276, May 1997.

OLIVEIRA, M. M. M.; TROCOLLI, B. T. Saber envelhecer. Principia, Paraíba, v. 4, n. 8, p. 1-7, set. 2002.

POLLOCK, M. L. et al. Twenty-year followup of aerobic power and body composition of older track athletes. Journal of Applied Physiology, v. 82, n. 5, p. 1508-1516, May 1997.

POWERS, S. K.; HOWLEY, E. T. Fisiologia do exercício: teoria e aplicação ao condicionamento e ao desempenho. 3. ed. São Paulo: Manole, 2000. 
ROBINSON, S. Experimental studies of physical fitness in relation to age. Arbeitsphysiologie, v. 10, s. n, p. 251-323, 1938.

SALTIN, B. Aging, health and exercise performance. Muncie: Ball State University, 1990.

WEINECK, J. Biologia do esporte. 7. ed. São Paulo: Manole, 2005.

WHO - World Health Organization. Health Evidence Network. What are the main risk factors for disability in old age and how can disability be prevented. Copenhagen: WHO Regional Office for Europe, 2004. 